INRA Prod. Anim.,

2014, 27 (2), 101-112

\title{
Elevage de précision en systèmes d'élevage peu intensifiés
}

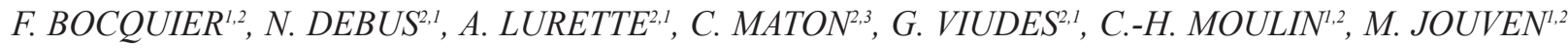 \\ ${ }^{1}$ Montpellier SupAgro, UMR868 Selmet, 2 Place Pierre Viala, F-34060 Montpellier, France \\ 2 INRA, UMR868 Selmet, 2 Place Pierre Viala, F-34060 Montpellier, France \\ ${ }^{3}$ Montpellier SupAgro, Domaine du Merle, Route d'Arles, F-13300 Salon de Provence, France
}

Courriel : françois.bocquier@supagro.inra.fr

L'élevage de précision connait un essor important en élevage intensif et en milieu confiné. Qu'en est-il des besoins et des perspectives de développement des techniques de précision pour l'élevage extensif, souvent caractérisé par la gestion d'un grand nombre d'animaux sur de grandes surfaces?

Comme pour l'agriculture de précision, le terme «élevage de précision » renvoie aux moyens mis en ouvre pour ajuster finement la conduite en tenant compte de la diversité des supports biologiques (animaux, végétaux) et de leurs réponses physiologiques et comportementales. Dans la mesure où diversité et réponses peuvent évoluer avec le temps, ces ajustements sont à concevoir dans un contexte dynamique. L'objectif de l'élevage de précision est de contribuer à améliorer le bien-être des animaux et l'impact environnemental de l'élevage tout en préservant les performances techniques et économiques des systèmes. Du point de vue de l'éleveur, la possibilité de diminuer la pénibilité des tâches est également un élément déterminant pour investir dans l'élevage de précision (Hostiou et al 2014, ce numéro). Par les conduites ajustées qu'il permet, en offrant de nombreuses possibilités de maîtriser les animaux en milieux ouverts, l'élevage de précision est un des moyens d'atteindre les objectifs d'une démarche agro-écologique (Jouven et al 2010, Dumont et al 2013).

Parmi les moyens mis en œuvre en élevage de précision, l'électronique embarquée au sens large, définie comme un dispositif placé sur l'animal, qui est autonome, fonctionnant souvent en temps réel, et spécialisé dans une tâche bien précise, est au centre des attentions. D'autres outils et méthodes peuvent être mobilisés dans ce but, et il existe aujourd'hui un large éventail de nouveautés technologiques qui apparaissent en centre de recherche (Brown et al 2013) ou sur le marché. Les supports de l'élevage de précision varient selon les espèces animales et les formes d'élevage associées. De très nombreux articles ont traité de technologies qui sont utilisables sur des animaux élevés en confinement (bovins laitiers, porcs et volailles) sur lesquels les moyens (financiers, temps, humains) développés peuvent être considérables (à cet égard le robot de traite qui mesure la composition du lait, le rythme cardiaque, la température corporelle et l'activité physique... est un exemple extrême) avec une très forte technicité des opérateurs (Wathes et al 2008, Jago et al 2013). A certains égards, le monitoring de ces animaux de très haute valeur économique s'orienterait surtout vers une maximisation des performances ; les informations recueillies permettant de mieux anticiper les décisions relatives à chaque animal et, dans ce sens, aider à optimiser la conduite générale du troupeau. Dans cet article, nous avons pris le parti de nous intéresser aux élevages peu intensifiés, c'est-à-dire présentant une faible production par animal et/ou par hectare, associée souvent à de grands troupeaux et à de vastes surfaces pastorales.

L'objectif de cet article est de proposer une réflexion sur la mise en œuvre de dispositifs innovants pour réaliser des actions de pilotage des animaux dans des systèmes d'élevage peu intensifiés tels qu'on peut en trouver en France et plus généralement en Europe. Comme pour l'ensemble des systèmes de productions, les solutions technologiques doivent être simples, efficaces et d'un coût acceptable en regard de la productivité des systèmes. La principale spécificité des dispositifs envisagés en systèmes peu intensifiés repose sur leur capacité à s'appliquer à des animaux dispersés sur des surfaces étendues. Sans ignorer que certaines réalisations mises au point en élevages intensifs pourraient être transférées vers des élevages plus extensifs, nous mettrons l'accent sur des dispositifs automatisés qui permettent de conserver une liberté de mouvement des animaux, qui s'appuient sur leurs facultés d'adaptation et qui pourraient améliorer le bien-être animal. Sont a priori exclus de cet article les dispositifs mécaniques automatisés utilisés en agriculture de précision pour les productions végétales (Abhijit et al 2013) y compris lorsqu'il s'agit de produire des aliments destinés aux animaux d'élevage (fourrages, céréales, oléo-protéagineux) ou encore de caractériser des végétations spontanées. En réalité certains d'entre eux pourraient également trouver des applications en élevage peu intensifiés.

\section{1 / Concepts généraux de l'élevage de précision : où se situent actuellement les inno- vations pour des formes d'élevage peu intensifiées?}

\section{1 / Définition de l'élevage de précision}

Un élevage de précision ( Precision Livestock Farming »-PLS) peut se définir comme un élevage dans lequel les opérations qui sont faites sur les animaux sont différenciées par unité du troupeau (individu ou lot), sur la base de mesures automatisées qui ont été transmises puis intégrées dans un Système d'Information (SI ; Jago et al 2013). Dans des élevages peu intensifiés de ruminants, l'enjeu technique porte principalement sur l'acquisition d'informations à l'échelle de l'animal dans de grands troupeaux souvent dispersés car pâturant de vastes surfaces. Ces informations portent généralement sur les caractéristiques physiques, 
le comportement physiologique, l'état sanitaire et les performances productives. Dans ce type de SI, les données acquises sont analysées puis transformées en vue d'une prise de décisions (par le système ou par l'éleveur) qui sont ensuite mises en œuvre en cohérence avec le système d'élevage. Ainsi, l'élevage de précision permet à la fois de caractériser la diversité au sein d'un troupeau via la mesure d'indicateurs à l'échelle de l'individu, mais aussi de mieux gérer cette diversité en proposant des indicateurs pour moduler la conduite à l'échelle des individus dans le but d'améliorer les performances du troupeau.

Dans les élevages qui n'ont pas recours à des dispositifs de précision, les actions ne sont individualisées que dans les situations critiques (mises bas, problème sanitaires...) et le traitement par lots d'effectifs importants, voire de tout le troupeau, est prépondérant. Dans cette dernière situation l'information individualisée y est généralement faible ou même absente. Nous excluons également du concept d'élevage de précision les élevages qui réalisent de nombreuses mesures sur les individus, mais qui n'utilisent pas ces mesures pour différencier les opérations de conduite entre individus. Toutefois lorsqu'une information individualisée existe, même si elle ne porte que sur l'observation d'animaux particuliers du troupeau (animaux " pilotes ») dont l'état ou le comportement va influencer la décision d'opérations de conduite pour l'ensemble du troupeau, nous sommes à nouveau dans une forme d'élevage de précision.
Au final, la qualification d'élevage de précision reste assez subjective. C'est ainsi que des pratiques d'élevage, pourtant classiques, qui individualisent les traitements à partir de l'observation attentive des animaux, sans recourir à des mesures objectives et automatisées devraient logiquement relever également de l'élevage de précision. En pratique, dans la littérature le terme « élevage de précision " est réservé aux situations où l'acquisition et/ou le traitement d'informations est automatisé. Dans le cadre de cet article, nous proposons de réserver cette appellation d'élevage de précision à des élevages comportant des effectifs importants d'animaux, faisant face à une disponibilité de main-d'œuvre réduite, pour lesquels les opérations de conduite sur des individus ou des lots d'un troupeau sont déclenchées en référence à des informations récentes traitées par le SI (figure 1).

\section{2 / L'évolution récente de l'éle- vage de ruminants présente des opportunités pour le développe- ment de l'élevage de précision}

Au cours de la décennie 2000-2010, en France le nombre d'exploitations avec un élevage herbivore significatif a continué à baisser de façon importante (-26\%), alors que le nombre d'UGB n'a diminué que de $7 \%$ (Perrot et al 2013). La taille du cheptel par exploitation a ainsi continué d'augmenter. Selon les espèces, l'augmentation du cheptel national par exploitation, en nombre de têtes, est de 11 à $116 \%$ sur la période $(+38 \%$ pour les vaches laitières, $+33 \%$ pour les

Figure 1. Représentation schématique des flux d'informations dans des systèmes d'élevage de précision.

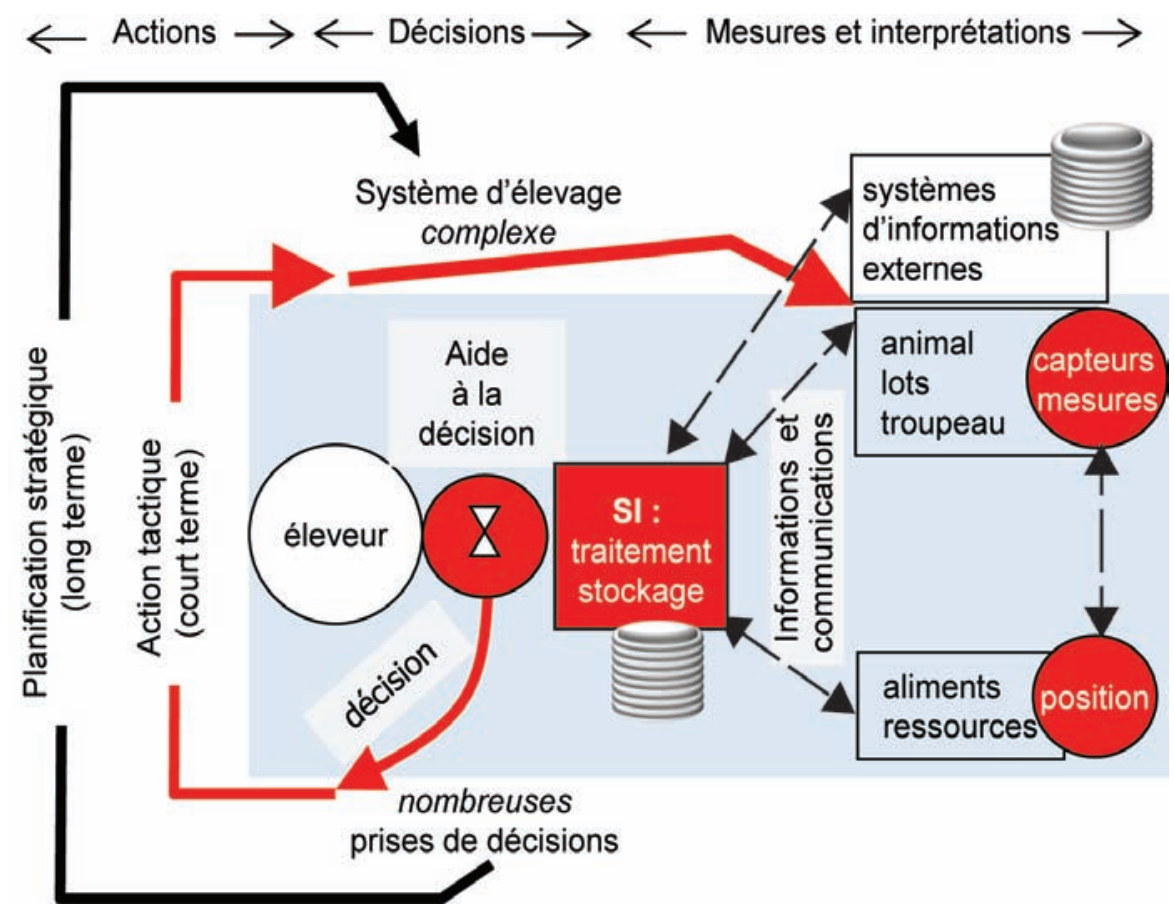

vaches allaitantes, $+116 \%$ pour les chèvres et $+42 \%$ pour les brebis à viande). En revanche, la taille des troupeaux dans les exploitations de brebis laitières, déjà importante en 2000, a peu augmenté avec des effectifs de 280 brebis (Agreste 2011). La proportion de troupeaux de grande taille a également augmenté, en général au-delà de $10 \%$. Dans ces exploitations de grande taille, selon la classification d'Agreste (2011), les effectifs de vaches laitières ont atteint en moyenne, en 2010, 102 vaches et pour les vaches allaitantes 113 vaches. Pour les petits ruminants, ils sont de 289 pour les chèvres laitières et de 346 pour les brebis allaitantes. En parallèle, le nombre d'Unités de Travail Annuel (UTA) par exploitation a également augmenté mais dans une proportion bien moindre (6\%, d'après RGA, Agreste). Ainsi globalement, à l'échelle française, le nombre d'animaux entretenus par UTA a augmenté. Ceci se confirme localement, avec par exemple une augmentation de $49 \%$ du nombre d'UGB par travailleur dans les 51 exploitations bovines du réseau Charolais suivi par l'INRA (Veysset et al 2013).

Pour réaliser cette augmentation de la productivité du travail, une simplification des pratiques peut être mise en place (Hostiou et Fagon 2012), visant à réduire le nombre d'interventions sur le troupeau. Cette simplification des pratiques (réduction du nombre de distributions d'aliments en bâtiment par exemple) ne remet pas forcément en cause une surveillance du troupeau et des interventions individuelles à des moments clés du cycle de production (mises bas, par exemple) ou pour réagir à des événements ponctuels. En parallèle, de nombreuses évolutions de l'environnement socioéconomique conduisent les éleveurs à "produire quantité de papiers à destination de tiers, du fait des exigences de traçabilité réglementaires et contractuelles » (Joly et Gautier 2006). Ces exigences de traçabilité se traduisent souvent par des informations transmises à l'échelle de l'animal, grâce à l'identification individuelle. Ainsi, la réforme de l'identification et de la traçabilité des petits ruminants a mis en place l'identification individuelle en 2005, la traçabilité par lot en 2009 et le suivi individuel des mouvements d'animaux en 2012 (avec dérogation pour les animaux de moins de 12 mois destinés à être abattus en France). En conséquence, les éleveurs sont de plus en plus souvent amenés à gérer une multitude d'informations, souvent écrites (Havet et al 2005), entre celles qu'ils génèrent et utilisent pour la conduite de leur exploitation et celles qu'ils reçoivent ou doivent transmettre à des tiers (figure 1).

En élevages peu intensifiés, avec un niveau de production par animal ou par 
hectare faible, la rentabilité économique est intimement liée à la capacité des systèmes d'élevage à être très économes en intrants au sens large (aliments et engrais achetés, mécanisation, main-d'œuvre) tout en conservant un niveau de production correct. Cette économie d'intrants est permise notamment par un pilotage fin du système, qui s'appuie sur la diversité des supports biologiques et leurs capacités d'adaptation. Dans ces conditions, on peut imaginer qu'il soit intéressant pour l'éleveur de réduire sa charge mentale en confiant en partie l'acquisition et/ou la gestion des informations multiples et variées à un SI et de diminuer parallèlement, par des automatismes, les efforts physiques lors des manipulations des animaux ou des aliments avec une assistance mécanique automatisée. Les dispositifs mis en œuvre en élevage de précision ne visent pas à remplacer les connaissances des éleveurs, mais à i) les compléter avec des informations recueillies par différents types de capteurs, ii) contribuer à la prise de décision en produisant des indicateurs opérationnels et iii) faciliter la mise en œuvre d'une gestion individualisée (cas de dispositifs couplés à des portes de tri). Les informations recueillies par les capteurs devraient essentiellement permettre une identification des animaux, facilitant ainsi la pesée, le tri, et la gestion alimentaire et/ou sanitaire des troupeaux. De plus, la fiabilité des données recueillies peuvent participer à sécuriser l'éleveur dans sa prise de décision par le biais d'outils de diagnostic technique ou économique.

\section{3 / Etat actuel de l'électronique et des capteurs en élevage}

La liste exhaustive des dispositifs électroniques ou des capteurs utilisables en élevage (tous types d'élevages confondus) n'est pas possible à dresser tant ceuxci se sont diversifiés (Lai et al 2013, Aqeel-ur-Rehman et al 2014) et sans cesse renouvelés (Abhijit et al 2013). Ceci d'autant plus que dans de nombreuses situations pratiques, des capteurs détournés de leurs fonctionnalités spécifiques pourraient s'avérer suffisamment précis pour fournir un indicateur fiable sur l'état d'un animal donné (RopertCoudert et al 2012, Andriamandroso et al 2014). Banhazi et Black (2009) remarquent que les progrès les plus significatifs en la matière ont été obtenus par des développements originaux plutôt qu'en améliorant l'existant.

\section{a) La puce RFID : un support de choix pour l'élevage de précision}

Parmi les dispositifs électroniques placés sur les animaux, le plus courant est sans conteste la puce « Radio Frequency Identification » (RFID) destinée à l'iden- tification électronique des animaux (Eradus et Jansen 1999, Caja et al 2006 , Mercier et al 2006). Ces transpondeurs RFID sont des circuits électroniques passifs, qui sont activés par le champ magnétique induit produit par un appareil de lecture placé à proximité, qui renvoient un signal codant pour le numéro d'identité de l'animal. Ces dispositifs ont fait l'objet de très nombreux travaux pour finalement, en 1996, être définis par deux normes ISO 11784 et 11785 pour l'identification réglementaire des animaux d'élevage dont le but premier est la traçabilité sanitaire des animaux. Les transpondeurs sont programmés une fois pour toute en usine, ils n'ont donc pas la possibilité de stocker de l'information nouvelle. Ces identifiants sont destinés à faire le lien entre un animal et ses informations stockées dans une base de données centralisée. L'impossibilité d'écrire de nouvelles informations sur le transpondeur apparaît comme une limitation forte. Un nouveau standard est en train de se mettre en place (ISO 14223). Il conserverait les précédentes spécifications et y ajouterait un dispositif anticollision (transmission de tous les identifiants situés dans le champ à un instant donné) et surtout il prévoit la possibilité d'y inscrire de nouvelles informations. Ainsi, ces transpondeurs évolués devraient offrir une palette plus large de possibilités pour de futures applications en élevage. Les spécifications finales devraient être publiées en 2015.

Les supports de ces puces électroniques sont très variés tant dans leurs dimensions (pour s'adapter aux différentes espèces) que dans leurs formes (selon leur localisation sur ou dans l'animal). On trouve majoritairement ces transpondeurs intégrés dans les boucles auriculaires, assez proches du modèle classique en plastique, pour l'identification des bovins, ovins et caprins. Ces boucles présentent l'intérêt d'être visibles même si elles peuvent se perdre (Mercier et al 2006) et risquent d'être falsifiées. On trouve également ces puces insérées dans des bolus ruminaux (bâtonnet de céramique séjournant de façon permanente dans le rumen), qui présentent l'avantage d'avoir peu de pertes, d'être infalsifiables mais qui nécessitent des lecteurs plus puissants (Caja et al 2006). Enfin, il existe des puces incluses dans du verre qui sont injectables sous la peau. Ces dernières sont réservées aux équins et aux animaux de compagnie. De façon plus marginale on peut trouver des puces RFID intégrées dans des bracelets placés au niveau du paturon des pattes arrières chez les chèvres (Duroy et al 2009). Les règlements en vigueur dans les différents pays reposent sur des choix réglementaires qui sont, par exemple, pour la France les boucles électroniques et pour l'Espagne les bolus ruminaux, étant entendu qu'ils sont tous lisibles par les lecteurs agréés. A ce jour, en France, toutes les populations animales d'élevage ne sont pas systématiquement identifiées électroniquement. Une réglementation européenne a rendu obligatoire une identification électronique systématique des ovins et des caprins pour fin 2014 (Mercier et al 2006).

Ces puces RFID ne sont pas de véritables capteurs mais sont porteuses d'une information essentielle qui est l'identité de l'animal. Elles sont suivies dans la chaine de traçabilité grâce à des lecteurs répartis des lieux de vente d'animaux aux abattoirs. En revanche, en élevage, le déploiement de ces technologies ne s'est pas accompagné de la fourniture de lecteurs RFID, qu'il s'agisse de lecteurs portables (« handheld») ou de lecteurs fixes («stationnary») (Mercier et al 2006). Il en résulte que les éleveurs ne sont pas toujours convaincus de l'intérêt de ces technologies, qui ne servent qu'au moment des inventaires annuels ou des contrôles de performances (contrôle de croissance, contrôle laitier) réalisés par les techniciens des structures d'appui. Ce qui prend du sens en élevage de précision c'est le moment et le lieu où se fait la lecture de cet identifiant. La lecture est alors porteuse d'une information de localisation qui peut être précieuse : dans ce cas c'est le lecteur RFID qui est le capteur.

Les lecteurs RFID sont des capteurs dits intelligents : ils stockent et traitent l'information acquise. En effet ils comportent de plus en plus souvent un système de stockage de données (mémoire), des algorithmes de vérification de la cohérence de ces données (inventaires) et sont programmables au point de constituer un carnet électronique de l'éleveur avec lequel il est possible de collecter des données au moment des mises bas (Carnet de Bergerie Informatisé : CBI ou Vénus) sur des animaux isolés ou encore de créer des listes d'animaux sur lesquels une action doit être entreprise. Ainsi, comme à tout moment il est possible de saisir une information et/ou de prendre connaissance de l'histoire de cet animal, c'est incontestablement un maillon d'un élevage de précision. Le principal intérêt de ces lecteurs est d'acquérir très rapidement l'information avec un taux quasiment nul d'erreur (i.e. réglementairement $<1 \%$ ), contrairement aux lectures visuelles qui sont souvent entachées d'erreurs de lecture ou de saisie. Des applications existent déjà et sont commercialisées. Elles permettent surtout l'identification électronique des animaux, facilitent la pesée, le tri, et la gestion des troupeaux par des logiciels. Mais d'autres applications sont possibles notamment dans le suivi sanitaire et physiologique des animaux. 


\section{b) Autres supports: GPS, biocapteurs, etc.}

D'autres composants électroniques, qui ont été développés et produits en masse, ces dernières années pourraient servir de capteurs en élevage (figure 2). C'est le cas des récepteurs de géo-localisation existants (GPS) qui peuvent être couplés à des Systèmes d'Information Géographique (SIG). Une des fonctionnalités qui a été imaginée, en dehors des systèmes classiques de suivis de mobiles, pour la certification géographique sera présentée ci-après. On peut également citer les accéléromètres qui ont été suffisamment miniaturisés pour être intégrés dans des dispositifs portés par les animaux d'élevage et suivre ainsi leurs activités motrices ou alimentaires. Enfin, il y a la génération des biocapteurs, c'est-à-dire ceux qui peuvent coupler des réactions biochimiques à des supports électroniques permettant d'envisager des mesures de paramètres biologiques tels que les taux sanguins de métabolites ou d'hormones, mais ils ne semblent pas s'être développés à ce jour (Velasco-Garcia et Mottram 2003, Ropert-Coudert et al 2012).

Ces capteurs, ayant acquis de l'information sur l'animal, doivent impérativement être associés à des moyens de transmission des données à distance avec pour objectif de limiter les interventions humaines. A ce stade de la description des systèmes existants on peut les différencier selon les distances qui séparent l'émetteur du récepteur (Mercier et al 2006, Aqeel-ur-Rehman et al 2014). A courte distance, quelques mètres, les technologies de type Bluetooth $\AA$ ou Zigbee $\AA$ sont utilisables pour faire communiquer les capteurs avec des récepteurs et/ou des automates. Ces technologies permettent de s'affranchir des connections filaires qui risquent d'être arrachées par les animaux. Ils assurent surtout à l'opérateur une grande liberté de mouvement. Pour des plus grandes distances, la centaine de mètres, les transmissions par Wi-Fi® sont les plus adaptées. Enfin à très grande distance, kilométrique, on peut recourir aux technologies éprouvées qui sont utilisées en téléphonie. La couverture par un réseau téléphonique fiable est alors nécessaire ce qui n'est pas toujours le cas. Il est à noter que les obstacles à la propagation des ondes (cloisons métalliques, murs, toitures, relief) et la nécessité de disposer d'une source d'énergie (électricité) sont des contraintes fortes au déploiement de ces technologies dans des milieux isolés et/ou difficiles. Enfin, il faut envisager dans la plupart des cas une organisation des transmissions des informations en réseau local (Lai et al 2013, Aqeel-urRehman et al 2014), ce qui permet d'assurer la couverture de la zone sous contrôle et de pouvoir à tout moment recueillir
Figure 2. Représentation schématique de différents capteurs susceptibles d'être embarqués sur des animaux d'élevage.

La double flèche du bas, figure le cheminement de l'information depuis le stockage et/ou vers un dispositif de transfert à distance (d'après Lai et al 2013).

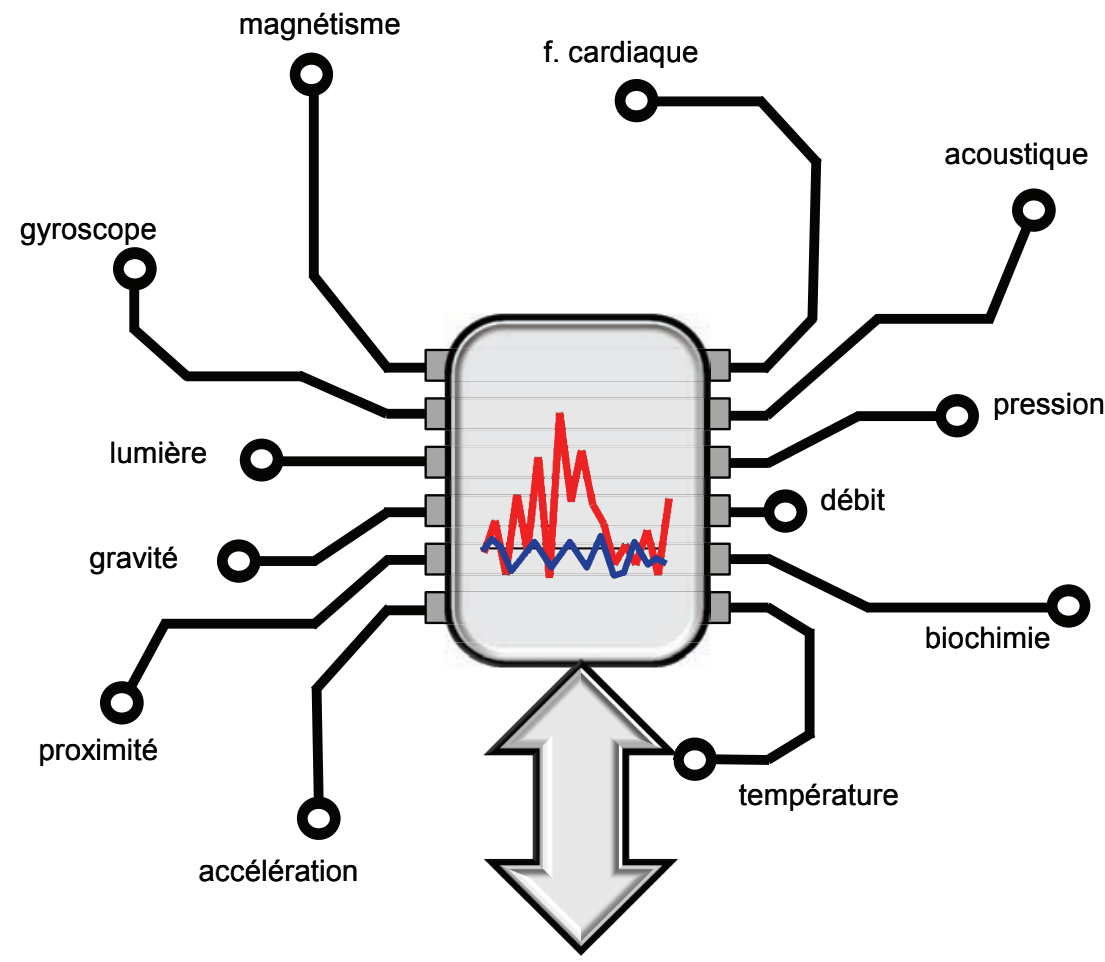

des données et/ou appliquer des actions (fermeture à distance d'une barrière, mise en route d'une éolienne par exemple). Ce type d'approche est déjà utilisé en Australie (《SmartFarming », voir www. csiro.au), pour localiser, par triangulation, les bovins à $7 \mathrm{~km}$ de distance avec une précision de $15 \mathrm{~m}$ (Taggle ${ }^{\mathrm{TM}}$ sensor). Les applications sont la localisation pour connaître les habitudes des animaux ou l'alerte lors de sortie de la zone en raison d'un vol d'animaux ou d'une attaque de prédateur.

\section{4 / Les contraintes techniques de l'élevage de précision en éle- vages peu intensifiés}

Le développement de l'élevage de précision en productions intensives a été facilité par les conditions d'élevage en bâtiment et par la logique de conduite visant à créer artificiellement des conditions de milieu optimales. Les automatismes sont entrés très tôt en élevages de porcs ou de volailles tout d'abord pour la régulation de l'ambiance des bâtiments (de type domotique). N'étant limité ni par les sources d'énergie et ni par les intempéries, il a ensuite été possible de transférer ces moyens développés dans l'industrie (agroalimentaire par exemple) pour proposer des systèmes d'alerte (température, humidité, luminosité, détecteur de mouvement, de proximité) ou d'observation directe des animaux (vidéosurveillance) et même l'utilisation de chariots autonomes de distribution d'aliments (Banhazi et Black 2009). Chez la vache laitière, la présence des animaux à la traite deux fois par jour permet de vérifier l'état des animaux et de réaliser des mesures liées à la production (volume de lait, cinétique d'émission du lait) ou même de disposer de moyens techniques plus lourds (analyse en temps réel de composition du lait, du $\mathrm{pH}$ et du nombre de cellules) puisqu'ils sont implantés à un seul endroit et peuvent constituer une annexe technique dédiés aux mesures sur les animaux pendant la traite (Jago et al 2013). La traite, pendant que les animaux sont immobilisés, peut être le lieu de téléchargement des données accumulées par les capteurs portés par l'animal. On peut comprendre que les deux situations d'élevage décrites cidessus sont plutôt favorables au développement d'un élevage de précision tel que l'on le rencontre maintenant dans des pays industrialisés (Wathes et al 2008, Jago et al 2013).

Les élevages peu intensifiés concernent surtout les herbivores conduits en majorité au pâturage et qui utilisent peu ou pas de bâtiments. Les infrastructures mobilisées sont souvent assez limitées. En simplifiant, on peut considérer qu'une des caractéristiques des élevages peu intensifiés est de reposer sur des nombres importants d'animaux assez peu productifs et rustiques. Dans la plupart des cas ces animaux utilisent de vastes surfaces 
qu'ils pâturent en parcourant quotidiennement de grandes distances (Jouven et al 2010). Dans ces conditions, assez différentes de celles rencontrées en élevages intensifs, les besoins des éleveurs pour les technologies de précision restent à identifier. On peut penser que le développement de l'élevage de précision sera envisagé en priorité pour la gestion des phases du cycle de production perçues comme critiques. La reproduction est un exemple de phase critique puisque sa réussite compte pour beaucoup dans la productivité animale. La réussite de la reproduction repose notamment sur la détermination du moment optimal de mise à la reproduction ou d'insémination artificielle grâce par exemple à la détection des chaleurs ; cet enjeu devrait donner lieu à des développements technologiques. Un autre élément central des élevages peu intensifiés est la contention et le tri des animaux au sein de grands troupeaux, qui déterminent la productivité du travail. Les systèmes de contention et de tri des animaux peuvent être couplés à des dispositifs motorisés et automatisés grâce à l'identification électronique. On peut également considérer que la conduite des animaux au pâturage peut être une contrainte forte lorsque le calendrier de travail est chargé. En particulier, dans des milieux ouverts et peu anthropisés l'absence de clôtures (fixes ou mobiles) entraîne la nécessité de garder les animaux, pratique contraignante alors que la main-d'œuvre devient un facteur limitant. Enfin, une autre des tâches essentielles dans ces élevages est liée aux traitements antiparasitaires qui s'imposent lorsque les animaux pâturent, mais dont la systématisation pose des problèmes sanitaires et environnementaux. De cette liste, somme toute incomplète, il apparaît que l'essentiel des besoins d'acquisition d'information pour la mise en œuvre d'un élevage de précision en systèmes peu intensifiés reposerait sur des données ponctuelles, transmises de manière discontinue, et visant à différencier les interventions par groupes d'individus au sein du troupeau.

Une contrainte générale en élevage de précision, qui est souvent signalée pour les élevages intensifs où l'acquisition d'informations est faite en continue et génère un volume très important de données, est la nécessité de maîtriser les flux d'information issus de ces capteurs. Il faut alors différencier l'information brute de l'information élaborée. Pour l'information brute, issue des capteurs, il faut impérativement des systèmes d'acquisition automatisés pour libérer totalement l'éleveur de cette tâche. Il faut ensuite que l'information brute recueillie, une fois élaborée ait une valeur zootechnique : qu'elle soit pertinente, fiable et si besoin répétée pour en saisir les évolutions. Il faut aussi que le dispositif d'acquisition soit simple à mettre en œuvre, facile à contrôler et capable d'autodiagnostic. Le coût de la mise en œuvre doit également être en rapport avec les services attendus. Ceci constitue une liste non exhaustive de conditions suffisantes. Les conditions nécessaires sont que le traitement de ces données brutes soit assuré par des algorithmes éprouvés délivrant une information élaborée qui soit fidèle aux situations rencontrées (Lai et al 2013). Au plan ergonomique, la représentation de la situation se doit d'être claire, de type alerte, et pouvoir donner lieu à une prise de décision rapide par l'éleveur. Il est certain qu'une large part d'initiative doit être laissée aux éleveurs et aux opérateurs techniques pour que la mise en œuvre de ces technologies permette des conduites d'élevage très élaborées et adaptées à des contextes variés.

\section{2 / Les réalisations techniques en cours ; des exemples et les enseignements à en tirer}

\section{1 / Le tri automatisé des ani- maux grâce à l'identification électronique}

Les inventaires d'animaux sont grandement fiabilisés par l'identification électronique, en intégrant des antennes de lecture RFID dans les couloirs de circulation des animaux. Compte tenu de la vitesse de lecture permise par ces puces RFID (700 brebis en 20 minutes au Domaine SupAgro du Merle, à Salon de Provence), cette opération est assez rapide même si elle n'est pas fiable à $100 \%$ en raison de la perte des identifiants, des passages simultanés d'animaux ou de la mauvaise position de la puce par rapport à l'antenne. La présence d'un opérateur attentif n'étant pas suffisante, dans le cadre des transactions commerciales, les animaux sont souvent identifiés par des lecteurs manuels qui signalent la bonne lecture de chaque identifiant. Dans tous les cas, en dehors des contraintes d'investissement, les dispositifs de tri automatisés présentent l'avantage de ne pas mobiliser d'opérateur supplémentaire qui serait dans l'incapacité de lire rapidement le numéro de l'animal, de le chercher dans une liste et de désigner de son orientation dans le lot adéquat.

Le tri des animaux est une opération fastidieuse qui est pourtant à la base d'un élevage de précision dans les grands troupeaux. Les dispositifs mécaniques existent depuis longtemps et de nombreuses variantes sont proposées selon les espèces d'élevage. Cependant ces dispositifs, qui ont été conçus pour être actionnées par un ou plusieurs opérateurs, ne sont pas toujours efficaces lorsqu'ils sont automatisés. De nombreux modèles existent (entre autres à l'INRA de Bourges) et sont maintenant commercialisés. Nous avons travaillé à la conception de telles portes de tri pour les ovins (Maton et al 2006). Il ressort que, compte tenu du comportement grégaire des ovins, le flux d'animaux doit être régulier et, pour maintenir la cadence, une voie de retour vers le troupeau initial est nécessaire pour remettre dans le flux les animaux qui n'ont pas été correctement identifiés. Une autre alternative récente (Wallace ${ }^{\mathrm{TM}}$ ) consiste à déclencher un spray de peinture sur les animaux nonlus et à les laisser passer pour ensuite les identifier manuellement. Les enseignements que nous tirons de ces expériences de tri chez les ovins sont que les animaux dont l'identifiant reste difficile à lire (comportement, qualité du capteur...) réduisent la cadence et perturbent le processus de tri : il faut donc trouver des solutions pour les écarter rapidement et les traiter ultérieurement.

Dans le cadre des opérations de tri des animaux il paraitrait intéressant de mettre au point des portes de tri sélectives dont l'ouverture serait conditionnée à la correspondance au lot auquel l'animal est destiné (vente, interventions vétérinaires, besoins alimentaires plus importants...). Si de tels dispositifs ne sont pas contraints par le débit des animaux ils doivent pouvoir fonctionner en l'absence d'opérateurs et être basés sur la motivation des animaux à franchir cette porte (Laca 2009). Davantage que pour les portes de tri citées ci-dessus, il est nécessaire de réaliser un apprentissage des animaux qui peut s'avérer fastidieux dans un premier temps, mais qui lorsqu'il est acquis persiste (cf. clôtures virtuelles). Pour les opérations de tri, le SI engendre des listes informatiques utilisables directement ou nécessitant une analyse de l'information. Dans tous les cas, les tris des animaux se font sur la base de critères choisis par l'éleveur.

\section{2 / Interactions entre animaux, le cas du comportement sexuel}

Chez les ruminants d'élevage la détection des chaleurs, qui est la phase clé de la mise à la reproduction, repose classiquement sur l'observation des chevauchements, ou des marques de chevauchement, par un mâle (ovins, caprins) ou, éventuellement, par d'autres femelles (bovins). Cette méthode, basée sur l'observation, est fastidieuse (observation ou relevé des marquages deux fois par jour par l'éleveur) et imprécise (évaluation subjective par l'éleveur de l'intensité du marquage des croupes des femelles). Les tentatives d'automatisation de la détection de ce comportement 
ont donné lieu à un très grand nombre de dépôts de brevets surtout chez les bovins laitiers pour lesquels les coûts de l'infertilité sont extrêmement élevés (Esslemont et al 2003). Chez les petits ruminants le traitement systématique et simultané de toutes les femelles par des traitements hormonaux a été la solution adoptée pour réaliser l'insémination artificielle à horaire fixe (Pellicer-Rubio et al 2009). Cette solution, qui ne s'applique pas à tous les types d'élevage (interdite par exemple en élevage biologique) pourrait être remise en cause à l'avenir pour des questions de sécurité des aliments d'une part, et environnementales d'autre part, au même titre que les traitements allopathiques systématiques en élevage. Des solutions alternatives mobilisant l'élevage de précision sont donc à l'étude.

Parmi les dispositifs électroniques mis en œuvre dans les exploitations laitières intensives, citons des capteurs de pression placés sur la croupe des vaches $(\mathrm{DEC} \circledast$ : Saumande 2002, Heat Watch $\AA$ : Peralta et al 2005, Palmer et al 2010) qui donnent une indication sur le nombre et la fréquence des chevauchements, et des podomètres qui traduisent la variation de l'activité locomotrice des vaches en chaleurs tels que le Heatime ${ }^{\circledR}$ (Valenza et al 2012) ou le HeatPhone ${ }^{\circledR}$. Pour tous ces dispositifs, seules les femelles équipées peuvent être détectées, ce qui suppose une connaissance de leur stade physiologique précis et l'installation des appareils de mesure à la bonne période. En outre une source d'énergie embarquée est nécessaire sur les vaches équipées pour mesurer, analyser et transmettre les données à une base de réception. Par ailleurs, les podomètres proposés ne seraient pas, par principe, adaptés à des situations où l'activité physique varie fortement d'un jour sur l'autre. En effet, les signaux sont analysés par rapport à une situation de référence interne, ce qui suppose une certaine stabilité des conditions d'élevage, condition non validée en milieux peu intensifs.

Pour des élevages extensifs, le système proposé est assez différent puisque c'est l'identifiant électronique passif (sans source d'énergie) qui est placé dans la zone de la croupe chez toutes les femelles du troupeau (Bocquier 2004). Un mâle ne pouvant pas saillir (équipé d'un tablier ou vasectomisé) est équipé d'un lecteur RFID autonome (Alpha-R, Wallace ${ }^{\circledR}$ ) qui se déclenche à chaque chevauchement et qui lit automatiquement l'identifiant de la femelle chevauchée. Dans ce cas la proportion des animaux porteurs d'une source d'énergie est très faible puisque seuls quelques mâles sont équipés. Ce dispositif a été validé chez la brebis (Bocquier et al 2006, Maton et al 2010, Viudes et al 2012, Alhamada et al soumis). Pour cette espèce $93 \%$ des chevauchements sont accompagnés d'enregistrement de l'identifiant. Comme les chaleurs s'accompagnent de nombreux chevauchements c'est la totalité des brebis qui sont détectées. Le dispositif porté par le mâle permet de s'affranchir des problèmes de spécificité associés aux détecteurs développés chez les bovins. Les faux-positifs sont facilement repérés, ils correspondent à des chevauchements isolés. Ils peuvent ainsi être supprimés des enregistrements du détecteur lors de l'analyse des résultats (Alhamada et al soumis). Le travail de détection des chaleurs pour l'éleveur consiste donc à relever les informations de chevauchement contenues dans les détecteurs (Alpha-D) des béliers équipés. Avec un récepteur radio (Alpha-R), l'éleveur peut, jusqu'à une distance de $100 \mathrm{~m}$, relever les informations contenues dans les détecteurs Alpha-D. Pour prendre la décision de mettre les brebis à la reproduction il dispose d'informations d'aide à la décision à l'écran et utilise ensuite le récepteur radio Alpha-R comme interface avec un automate de tri des animaux ce qui lui permet d'isoler les femelles à mettre à la reproduction. Un tel dispositif devrait permettre de détecter les femelles vides (celles qui reviennent en chaleur), d'établir le planning des mises bas et également d'évaluer automatiquement la libido des mâles. Dans le cas de la mise en œuvre d'un effet mâle comme alternative à l'utilisation des traitements hormonaux (Pellicer-Rubio et al 2009), ce détecteur permettrait également de réaliser des inséminations si le nombre de femelles groupées est suffisant. Cet appareil fonctionne également chez la chèvre (figure 3 ).

\section{3 / La gestion du pâturage : comportement alimentaire et répartition spatiale}

Les situations où le pâturage est dominant sont souvent associées à des formes d'élevage peu intensifiées. Dans les systèmes avec des objectifs de production modérés, l'autonomie alimentaire (intimement liée à la performance globale de l'exploitation) dépend directement de la capacité à ajuster l'utilisation d'une diversité de ressources pâturées (souvent spontanées) par un troupeau hétérogène. Cet ajustement a pour but d'une part, d'offrir aux animaux une alimentation pâturée conforme à leurs besoins (corrigés par les objectifs de production et pouvant inclure une mobilisation des capacités adaptatives) et, d'autre part, d'assurer une répartition spatiale du pâturage favorable au renouvellement de la ressource. Dans des systèmes avec des objectifs de production plus élevés, le pâturage a été longtemps délaissé tant les paramètres qui conditionnent les performances des animaux et l'entretien du couvert végétal sont imbriqués de façon complexe et donc difficiles à maîtriser. Le pâturage tend à être réintroduit aujourd'hui, car il reste le mode d'alimentation le plus avantageux du point de vue économique et environnemental. Ainsi, la conception d'outils d'aide au pilotage du pâturage permettrait d'une part, de réintroduire l'alimentation au pâturage dans les

Figure 3. Utilisation du détecteur de chaleur chez quatre chèvres synchronisées par traitement hormonal (Guillouet et Bocquier, non publié). Courbes de couleurs, nombre cumulé de chevauchements par chèvre au cours du temps. Courbe grise, le bouc change de chèvre. La ligne pointillée verticale représente le passage du jour à la nuit.

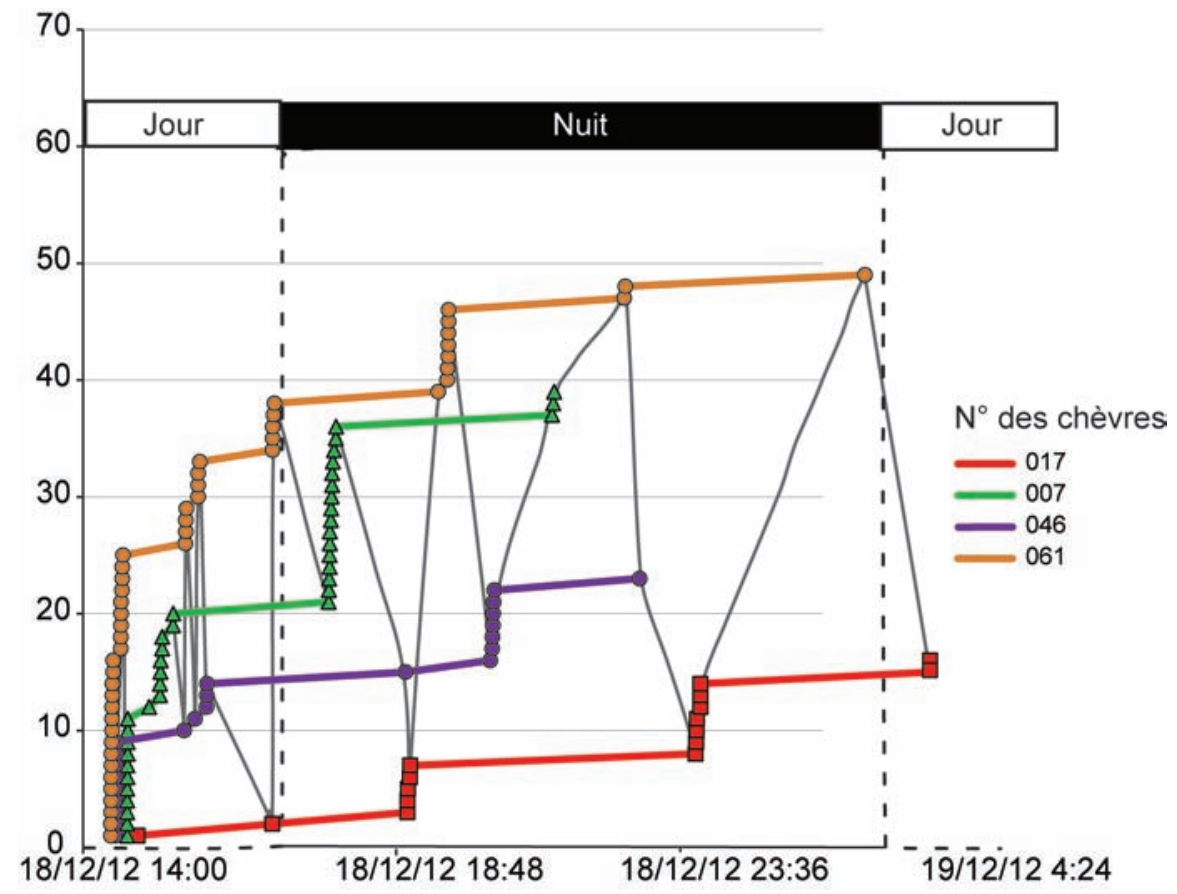


systèmes d'élevages intensifiés et, d'autre part, de sécuriser les performances zootechniques et écologiques dans des systèmes peu intensifiés.

Une des premières applications de systèmes électroniques embarqués, basée sur un capteur de tension placé au niveau des mâchoires, a été l'étude du comportement alimentaire des ruminants en station de recherche (Léveillé et al 1979). De tels systèmes, et ceux qui ont suivi, ne pouvant équiper tous les animaux d'un élevage, pour des questions d'autonomie énergétique et de solidité, ils sont restés cantonnés à la recherche. Ils ne peuvent donc pas, dans leur forme actuelle, être utilisés en routine dans les élevages où les animaux pâturent la plupart du temps.

Les enjeux liés à la maîtrise du pâturage sont tels, qu'un concept spécifique est proposé : le pâturage de précision (Laca 2009). Ce concept pourrait s'appliquer autant sur prairies que sur parcours. Le pâturage de précision repose sur les mêmes principes que ceux édictés précédemment pour l'élevage de précision, sauf qu'il cumule les difficultés d'estimer non seulement l'état des animaux mais également celui de la ressource alimentaire dans des milieux ouverts et vastes (question non abordée dans cet article). Mettre en œuvre un pâturage de précision permettrait d'établir des systèmes d'élevage durables, écologiquement intensifs (Dumont et al 2013). L'enjeu est d'ajuster, au fil du temps et en fonction des aléas, la diversité des ressources alimentaires offertes au pâturage à la diversité des besoins du troupeau. Le but ultime est de valoriser la très forte hétérogénéité spatiale et temporelle des couverts spontanés pour parvenir à des performances animales et environnementales élevées. La base d'un système de pâturage de précision repose sur une connaissance approfondie du comportement alimentaire des animaux et des dynamiques inter- et intra-annuelles des végétations pâturées.

On dispose maintenant de suffisamment de connaissances sur le comportement animal et le déterminisme des choix alimentaires pour mettre en œuvre des situations d'apprentissage ou de conditionnement opérants visant à orienter les déplacements et les comportements alimentaires des animaux (Provenza et Villalba 2006, Laca 2009). Néanmoins, la solution fréquemment retenue est de disposer d'attracteurs (lieu d'abreuvement, dépôt de pierres à sel, distribution d'aliments concentrés ou de fourrages appétents) pour modifier la répartition spatiale de la pression de pâturage dans de vastes espaces clôturés (Ganskopp 2001, Bailey 2005). Une solution alternative, basée sur des dispositifs électro- niques, est représentée par les clôtures virtuelles. Une clôture virtuelle peut être définie comme une structure délimitant une zone (aire géographique, zone d'exclusion autour d'un animal...) sans clôture physique (Umstatter 2011). Différents prototypes de clôture virtuelle ont été développés durant les 20 dernières années par des laboratoires américains, australiens et français (Tiedemann et al 1999, Anderson 2006, Bishop-Hurley et al 2007, Monod et al 2009). Ces prototypes sont basés sur des dispositifs embarqués sur l'animal (colliers, " serre-tête ») délivrant des stimuli neutres (sonore, tactile) visant à modifier le déplacement de l'animal à l'approche de la frontière virtuelle délimitée par un champ électromagnétique ou par des coordonnées géographiques, puis des stimuli aversifs (choc électrique) en cas de franchissement. Tout comme pour la clôture électrique, les animaux doivent préalablement être soumis à un apprentissage visant à associer le stimulus neutre au comportement requis (faire demi-tour ou changer de direction) sans déclenchement du stimulus aversif. Les essais réalisés jusqu'ici sur bovins, caprins et ovins montrent que ces animaux d'élevage sont capables d'apprendre rapidement à respecter une clôture virtuelle. Cependant, les essais menés en n'équipant qu'une partie du troupeau montrent que la combinaison de l'attraction sociale et d'une végétation appétente en zone « interdite » réduit l'efficacité de contention de la clôture virtuelle (Jouven et al 2012). Ainsi, en particulier pour de grands troupeaux où il n'est pas envisageable d'équiper tous les individus, les clôtures virtuelles sont à considérer comme un moyen de modifier la répartition de la pression de pâturage et non comme un mode de contention à proprement parler. Le choix des animaux à équiper pourrait reposer sur plusieurs critères liés à leur comportement alimentaire et spatial ou à leur rang social dans le troupeau (Dumont et Boissy 2000). Au final, les clôtures virtuelles peuvent constituer une aide précieuse dans la mise en place d'un pâturage de précision, en particulier dans des milieux où la pose de clôtures physiques est difficile et lorsque le gardiennage n'est pas envisagé. Parmi les applications possibles, citons notamment : i) le "redécoupage " de trop grands parcs (50 ha et plus) dans le but de limiter les sur- ou sous-exploitation localisées, ii) la mise en défens temporaire de zones d'intérêt patrimonial dans des périodes de sensibilité, iii) l'accès différentiel au sein du troupeau à la diversité des végétations présentes selon les besoins nutritionnels des animaux. Pour l'instant, aucun dispositif de clôture virtuelle spécifique pour les élevages pâturants n'est disponible en version commerciale. Des essais en élevages mobilisant des dispositifs commercialisés pour les chiens sont programmés pour 2014 dans des systèmes caprins pastoraux pratiquant des lâchers dirigés sur parcours.

Des systèmes moins précis de pilotage du pâturage ont été proposés (RuizGarcia et Lunadei 2011). De tels dispositifs, dotés de moyens de transmission à longue distance peuvent envoyer des signaux d'alerte aux éleveurs. En effet, pour ce qui est des animaux, il est assez classique de considérer que les changements de comportement alimentaire (temps d'ingestion) et/ou spatiaux (repos prolongés ou agitation) signent une insuffisance des ressources pâturées. Pour mesurer ces évolutions on peut songer à utiliser des appareils qui collectent automatiquement le comportement alimentaire ou la localisation en temps réel des animaux. Une façon élégante de localiser les animaux peut être de placer de simples lecteurs RFID à des endroits stratégiques du parcours (zones de repos, points d'abreuvement). Pour passer au pilotage du pâturage il faut associer la détection de changements de comportement alimentaire à une modification de l'offre alimentaire au pâturage, par exemple en donnant accès à de nouvelles zones plus attractives du parcours, ou à des distributeurs d'aliments ou déclencher à distance des signaux de renforcement connus de ces animaux (signaux visuels ou sonores).

Dans les élevages très intensifs, des systèmes de Distribution Automatisée des aliments Concentrés (DAC) ont été mis en place depuis plusieurs dizaines d'années. Pour les élevages peu intensifiés, les réalisations ont été rendues compliquées par la mobilité des troupeaux au pâturage et dans les systèmes de petits ruminants, elles ont été freinées par les effectifs qui peuvent atteindre plusieurs centaines à plusieurs milliers d'animaux. Il serait intéressant dans de telles situations de mettre au point des portes de tri sélectives mobiles permettant aux animaux identifiés comme ayant des besoins spécifiques (brebis allaitant deux agneaux, brebis maigres, agneaux) d'accéder progressivement à des ressources alimentaires pâturées de meilleure qualité nutritionnelle. Par exemple, une zone de végétation particulièrement nutritive et appétente au sein d'un grand parc pourrait n'être rendue accessible qu'à ces animaux. Ainsi, la diversité de ressources alimentaires offerte au pâturage pourrait être adaptée aux besoins des animaux sans scinder le troupeau en plusieurs lots physiques, tout en limitant aussi la pression de pâturage sur une zone potentiellement sur-pâturée. Il n'existe pas à notre connaissance de tels systèmes dans le commerce. 


\section{4 / Le drogage de précision ou les traitements antiparasitaires ciblés}

Une des nécessités des élevages où les animaux sont conduits essentiellement au pâturage est d'administrer des traitements antiparasitaires. Dans le cadre d'un élevage de précision il faudrait que ces traitements soient faits à bon escient uniquement sur les animaux parasités et à la dose recommandée. Une solution proposée en Australie consiste à ne traiter que les animaux qui accusent une perte de poids sur un intervalle de temps donné. Pour cela une bascule électronique est intégrée à un couloir de contention où l'animal est identifié et pesé. Selon la variation de poids, l'animal est, ou non, traité. Malheureusement de tels dispositifs n'ont pas été évalués et semblent être restés au stade du prototype. Nous avons, quant à nous, testé l'effet d'une dose d'antihelminthique avec un dispositif de distribution qui délivre une quantité exactement proportionnelle au poids vif mesuré des brebis laitières, alors que la pratique habituelle est de délivrer une dose unique correspondant à la brebis la plus lourde du troupeau. La comparaison des deux modes d'administration avec le dispositif Dose-it (Wallace ${ }^{\mathrm{TM}}$ ), conduit à une économie de produit dérisoire $(15 € / 100$ brebis) et un temps de travail 6 fois plus important que le drogage classique au cornadis. La prévalence des infestations, mesurée un mois plus tard, était identique entre les deux lots (Hoste et Bocquier, non publié). Dans le cas présent, en l'absence d'estimation des effets des excédents de ces produits sur la survenue de résistance des parasites, la rentabilité immédiate n'est pas assurée. Ainsi, seule une démarche responsable peut justifier l'utilisation de ces dispositifs d'élevage de précision pour la mise en place d'un élevage plus respectueux de l'environnement et de la biodiversité («Green, Safe and Ethical», Martin et Kadokawa 2006), ou surtout la survenue de résistances (Stafford et al 2009). Actuellement, seules des dispositions contraignantes ou incitatives sont susceptibles de modifier les pratiques.

\section{5 / La géo-certification des modes d'élevage}

Les élevages intensifs en confinement ne posent pas de problème quant à la localisation géographique puisque des contrôles d'entrée et de sortie des animaux, tels qu'ils sont réalisés dans le cadre des procédures de traçabilité, sont suffisants pour en garantir l'origine géographique. En revanche pour les élevages pastoraux, surtout s'ils transhument, leur localisation peut être compliquée. Or, il existe d'ores et déjà des cahiers des charges qui stipulent que les animaux doivent avoir valorisé certains espaces régionaux ou que des primes environnementales ne seront versées que si les animaux ont pâturé des zones écologiques particulières (lutte contre l'incendie, pistes de ski). Quelle que soit la taille et le nombre d'animaux il n'est pas envisageable de les équiper individuellement d'un GPS en raison du coût élevé et d'une autonomie énergétique insuffisante. Pour ces élevages nous avons breveté un dispositif couplant identification électronique individuelle et GPS lui-même couplé à un lecteur RFID comportant des spécifications spatio-temporelles cryptées. L'opération de certification consiste à effectuer des inventaires géolocalisés à des intervalles de temps préalablement définis au cahier des charges (Bocquier 2006). La certification, ou les primes, sont effectives à l'issue de la saison de pâturage lorsque tous les animaux ont été contrôlés dans les périmètres préétablis. Depuis cette date des dispositifs dédiés (type Simvalley ${ }^{\mathrm{TM}}$ ) sont disponibles pour le suivi des personnes ( GeoFencing »). Il s'agit en réalité d'un GPS relié à de la téléphonie qui déclenche une alerte lorsque le mobile sort d'une zone prédéfinie. Nous ignorons si cette technologie est utilisable en élevage : elle pourrait en partie remplacer la clôture virtuelle dans des zones où les délimitations sont floues, mais elle nécessiterait la présence d'un opérateur (éleveur, berger...) sur place pour rediriger les animaux sur la zone autorisée.

\section{3 / Conditions pour l'utili- sation de ces innovations en élevages peu intensifiés}

Pour que se développe l'élevage de précision en systèmes extensifs les conditions sont radicalement différentes de celles observées en élevage intensif. En effet, en système intensif - par exemple porcin (Banhazi et Black 2009) - il est clairement mis en avant la notion de paquet technologique à transférer. Cette position est rendue possible par la forte intégration entre l'éleveur et son environnement technique et économique. C'est ainsi que les fabricants peuvent proposer des solutions clés en main avec plusieurs capteurs et un SI adapté. De plus, dans ces élevages, les facteurs limitants ou les points critiques sont souvent bien identifiés et les outils techniques ainsi que les objectifs à atteindre font déjà partie intégrante du pilotage (niveau de production, indices de consommation, rythmes de reproduction). Pour des élevages plus extensifs, les contraintes liées à la diversité des conditions de production excluent pratiquement le transfert de tels paquets technologiques. D'une part, parce que les éleveurs sont loin d'avoir la même perception de la hiérarchie des facteurs limitants à mâ̂triser (Lurette et al 2012) et, d'autre part, parce que les conditions d'acquisition des informations pertinentes sont extrêmement diversifiées : ce qui supposerait une offre matérielle et logicielle multiforme qui n'est pas disponible actuellement. Ainsi, l'offre lorsqu'elle existe, peut ne pas apparaître immédiatement valorisable ou se heurte à un SI qu'il faudrait adapter ce qui fait peser sur les éleveurs et les structures d'encadrement des adaptations délicates. Dans ce contexte, les équipementiers sont aussi face au dilemme de devoir soit développer des outils assez peu connectés aux SI (ce qui en réduit l'intérêt) soit de s'y impliquer et donc de supporter les charges de développement sans avoir de garanties de retour sur investissement. La solution ne pourra donc venir que des structures d'appui technique locales ou nationales. En concertation avec les éleveurs, ces structures sont les seules capables (investissements financiers et humains) d'adapter l'existant aux offres technologiques avant leur diffusion sur le terrain.

Les élevages peu intensifiés devraient être fortement utilisateurs d'information « de précision » pour repérer les animaux particulièrement efficaces dans la cadre des nouvelles conditions " écologiquement intensives $\gg$ de production, pour notamment optimiser l'alimentation (autonomie alimentaire...) et anticiper les risques sanitaires (maîtrise du parasitisme...). Un des aspects critiques de l'élevage de précision, en milieux peu intensifiés, est que le stockage des données dans un SI peut rapidement devenir problématique en raison des temps d'acheminement des données, de leur volume et de leur traitement. Il est sans doute préférable, selon les fonctions biologiques suivies, de ne maintenir qu'une partie des données automatiquement collectées sur des serveurs locaux (Ruiz-Garcia et Lunadei 2011) ou sur les appareils de mesure : c'est en particulier le cas des mesures contextualisées qui ne servent qu'à la conduite et/ou qui ne sont issues que d'animaux marqueurs. Pour produire des informations élaborées, il est nécessaire de s'appuyer sur des algorithmes de traitement des données. Ceux-ci, comme nous l'avons vu dans les exemples, doivent être simples et robustes. Pour cela, ils acquièrent leur pertinence souvent par les itérations et variations relatives des signaux dans les situations les plus complexes. Par exemple, dans le cas du dispositif de détection des chaleurs, un algorithme permettant de sélectionner les brebis à mettre à la reproduction doit être adapté en fonction de la conduite du troupeau et notamment du temps de présence des mâles au contact des femelles. La construction de ces algorithmes sou- 
lève des questions sur la nécessaire normalisation des mesures et des traitements, si une telle information élaborée devait servir au phénotyage des animaux. Malgré la tendance des logiciels du commerce à fournir une « réponse automatique intégrée ", il faudrait favoriser les analyses de l'information faites par les producteurs car rien ne remplace vraiment le « tour de plaine » de l'agriculteur et l' « œil de l'éleveur » sur son troupeau, en particulier pour des systèmes complexes que sont les systèmes peu intensifiés basés sur le pâturage.

Il semble acquis que l'agriculture et l'élevage deviennent des champs d'application de plus en plus importants des Technologies de l'Information et de la Communication (TIC). Pour autant, seules les informations élaborées, obtenues de façon exhaustive qui ont été traitées et validées, méritent de rejoindre des SI centralisés. Les systèmes nationaux contiennent d'ailleurs bien souvent des informations qui sont peu ou pas valorisées pour le pilotage de l'élevage. Ainsi, à l'échelle collective (organisation de producteurs, filières de qualité, organismes de sélection et de contrôle de performances), la mise en place d'un élevage de précision devrait avoir pour objectif d'intégrer les nouvelles données et de valoriser celles qui sont déjà collectées (généalogies, contrôles de performances). La gestion des données reste un enjeu majeur. En effet, comme cela a été observé en élevage intensif (bovins laitiers, porcins), on peut craindre l'intervention de structures privées qui captent et valorisent les données sans les restituer aux structures classiques d'appui technique. Bien que des initiatives aient été prises en matière de normalisation des échanges de données, il subsiste une forte hétérogénéité matérielle et logicielle qui freine le développement de ces technologies car les différents matériels développés restent incompatibles.

\section{Conclusions et perspectives}

Le contexte actuel de l'élevage peu intensifié en France et plus généralement en Europe (grands troupeaux sur de grandes surfaces, avec peu de main-d'œuvre disponible), combiné à la généralisation de l'identification électronique des animaux, pose un cadre favorable au développement des technologies de précision. Pour des élevages comportant plusieurs centaines d'individus dispersés sur de grandes surfaces, avec des niveaux de production modérés à faibles, les besoins précis en élevage de précision restent à déterminer. Les domaines d'application possibles sont vastes, et incluent notamment le tri automatisé des animaux, la détection automatisée des chaleurs, le contrôle de la répartition des animaux au pâturage via des clôtures « virtuelles » ou encore la géo-certification des modes d'élevage.

Le développement de l'élevage de précision en systèmes peu intensifiés pose encore des questions techniques et organisationnelles, notamment sur la nature des données à stocker, leurs transformations par des algorithmes et les modalités d'utilisation par les éleveurs, mais aussi par des structures collectives dans le but de faire évoluer des races animales ou des modes de production. En particulier, l'élevage de précision en milieux difficiles est largement dépendant d'une bonne connaissance du milieu (parcours, pâturage, zones de repos, lieu d'abreuvement) et des animaux marqueurs (leaders - subordonnés, stades physiologiques particuliers, âges, races) ; connaissance qui est généralement détenue par les éleveurs et les techniciens. Une bonne connaissance du milieu et du fonctionnement du troupeau dans ce milieu permet de limiter l'implantation des capteurs aux seuls lieux pertinents et aux seuls animaux représentatifs. Dans ce contexte, les outils d'aide à la décision doivent eux aussi être faciles à paramétrer pour générer des alertes contextualisées. Pour d'autres actions à piloter, il faudrait parvenir à réduire le coût des capteurs pour que tous les animaux soient inclus dans les processus de contrôle ; c'est notamment le cas pour le suivi de la reproduction, l'historique des traitements vétérinaires, etc. Le but étant dans la plupart des cas de trier des animaux ayant des caractéristiques communes pour leur appliquer une conduite adaptée. Mais, dans ce type d'élevage de précision, il faudra aussi accepter que l'information soit assez hétérogène et uniquement utilisable pour la prise de décision. C'est en particulier le cas pour les outils d'alerte en cas d'attaque de prédateurs (loup, chien errant), de vols d'animaux ou tout simplement d'absence d'animaux à un point d'abreuvement. Enfin, le développement de SI collectifs constitue un enjeu qui pourrait s'avérer crucial pour la maîtrise des évolutions des systèmes d'élevage. Il existe donc plusieurs types d'outils mobilisables pour l'élevage de précision, qui répondent à des objectifs différents, à l'échelle de l'exploitation et/ou à des échelles plus vastes (région, filière, pays...). Il faudrait veiller à ce que les solutions retenues soient suffisamment ouvertes pour répondre aux besoins des acteurs des filières.

Bien que des outils existent déjà pour mettre en œuvre un pâturage de précision, la principale difficulté d'application en élevage réside dans la maîtrise du comportement animal. La conséquence est qu'il faut, dès la conception, intégrer régulièrement des phases d'apprentissage et de renforcements des comportements. Cet investissement nécessite du temps qui n'est pas perçu comme rentable tant que le système n'est pas opérationnel. La modélisation du comportement animal prend une importance fondamentale dans la compréhension et la conception de dispositifs permettant la maitrise du comportement des animaux. De tels dispositifs devraient en outre contribuer favorablement au bienêtre animal dans la mesure où ils s'appuient essentiellement sur les registres comportementaux innés de ces animaux. Cela amènerait à intégrer ces capteurs et effecteurs dans les nouveaux dispositifs expérimentaux, comme cela a été réalisé chez les animaux sauvages (revue de Brown et al 2013), avec pour conséquence de générer des connaissances fondamentales (réseaux sociaux) et appliquées (conduites innovantes).

S'agissant des matériels dédiés à un élevage de précision en milieux peu intensifiés il serait souhaitable que les professionnels se dotent d'une plateforme technique d'évaluation des matériels tant pour leur efficacité que pour la coordination des transferts d'information entre les dispositifs existants ou à venir. Cette plateforme pourrait aussi être un lieu de formation pour les éleveurs, techniciens et ingénieurs afin qu'ils puissent s'investir dans cette voie qui paraît prometteuse.

Remerciements : Nos travaux ont été soutenus depuis plusieurs années par différents financements : Projets Incitatifs de Département PHASE (INRA), Projet e-Mate (INRA, DGER), Projet Bercail (Transfert-LR, FEDER), Projet ReproBio (Casdar), ANR PANURGE. Nous remercions les personnes de l'Unité Expérimentale de Fréjorgues (J.-M. Capron), du Domaine du Merle (P.-M. Bouquet, J.-D. Guyonneau et D. Montier) ainsi que le Service Elevage de la Confédération de Roquefort et UNOTEC. Nous sommes reconnaissants à $D$. Santo, de la société WALLACE (143, ZI les Mourgues. 30350 Cardet, France), pour s'être investi dans ces développements. 


\section{Références}

Abhijit S., Nitaigour M., Kiseon K., 2013. A review on application of technology systems, standards and interfaces for agriculture and food sector. Comp. Stand. Interf., 35, 355-364.

Agreste, 2011. Agreste Primeur : structure des exploitations agricoles. www.agreste.agriculture.gouv.fr/IMG/pdf primeur272-2.pdf

Alhamada M., Debus N., Lurette A., Viudes G., Bocquier F., Electronic estrus detection in sheep as an alternative to visual observation. Small Rum. Res., (soumis).

Anderson D.M., 2006. Virtual fencing - a concept into reality. In: Proc. Spatial Grazing Behav. Workshop. Bishop-Hurley G.J. (Ed), 61-91.

Andriamandroso A.L.H., Lebeau F., Bindelle J., 2014. Accurate monitoring of the rumination behaviour of cattle using IMU signals from a mobile device. 2014. In: Proc. Eur. Grassland Fed., Aberystwyth University, 7 - 11 September 2014 (soumis)

Aqeel-ur-Rehman., Abbasi A.Z., Islam N., Shaikh Z.A., 2014. A review of wireless sensors and networks' applications in agriculture. Comp. Stand. Interf., 36, 263-270.

Bailey D.W., 2005. Identification and creation of optimum habitat conditions for livestock. Rangeland Ecol. Manag. 58, 109-118.

Banhazi T.M., Black J.L., 2009. Precision livestock farming: A suite of electronic systems to ensure the application of best practice management on livestock farms. Austral. J. Multidisciplinary Eng., 7, 1-14.

Bishop-Hurley G.J., Swain D.L., Anderson D.M., Sikka P., Crossman C., Corke P., 2007. Virtual fencing applications: implementing and testing an automated cattle control system. Comp. Electron. Agric., 56, 14-22.

Bocquier F., 2004. Method and device for automatically detecting mating of animals. 21.07.2005. WO/2005/065574, SupAgro, INRA Licence signée avec WALLACE, (Cardet, France) Novembre 2012.

Bocquier F., 2006. Device and method for spatially and temporarily counting animals. WO/2006/111680, 2006, INRA, SupAgro, CIRAD. Fin du soutien de l'INRA en Mars 2013.

Bocquier F., Gaubert J.L., Blanc F., Viudes G., Maton C., Debus N., Teyssier J., 2006. Utilisation de l'identification électronique pour la détection automatisée du comportement sexuel chez les ovins : perspectives pour la détection des chaleurs chez la brebis. Renc. Rech. Rum., 13, 155-158.

Brown D.D., Kays R., Wikelski M., Wilson R., Klimley A.P., 2013. Observing the unwatchable through acceleration logging of animal behavior: review. Anim. Biotelemetry 1, 1-16.

Caja J., Ghirardi J.J., Hernandez-Jover M., Bocquier F., 2006. Utilisation des bolus électroniques pour la traçabilité des ruminants : état de la technique, mise en place et évaluation en ovins et bovins. Renc. Rech. Rum., 13, 163 166.

Dumont B., Boissy A., 2000. Grazing behaviour of sheep in a situation of conflict between feeding and social motivations. Behav. Process., 49, 131-138

Dumont B., Fortun-Lamothe L., Jouven M., Thomas M., Tichit M., 2013. Prospects from agroecology and industrial ecology for animal production in the $21^{\text {st }}$ century. Animal, 7,1028 1043.

Duroy S., Holtz J., Bavary B., Mercier L. Marguin L., 2009. Conditions préalables à l'utilisation à grande échelle de l'identification électronique des bovins et des petits ruminants. Renc. Rech. Rum., 16, 200.

Eradus W.J., Jansen M.B., 1999. Animal identification and monitoring. Comp. Electron. Agric., 24, 91-98.

Esslemont R. J., Kossaibati M. A., Allcock J., 2003. Improving fertility in dairy cattle: the costs and the benefits. Proc. Soc. Feed Technol., $1-11$.

Ganskopp D., 2001. Manipulating cattle distribution with salt and water in large arid land pastures: a GPS/GIS assessment. Appl. Anim. Behav., Sci. 73, 251-262.

Havet A., Joly N., Cochet A., 2005. Les écrits comme supports de raisonnement dans les exploitations d'élevage. Renc. Rech. Rum., 12, 327-330.

Hostiou N., Fagon J., 2012. Simplification des conduits d'élevage : analyse transversale des pratiques mises en œuvre dans les filières herbivores et granivores. In : Numéro spécial, Travail en élevage. Hostiou N., Dedieu B., Baumont R. (Eds). INRA Prod. Anim., 25, 127-140.

Hostiou N., Allain C., Chauvat S., Turlot A., Pineau C., Fagon J., 2014. L'élevage de précision : quelles conséquences pour le travail des éleveurs? In : Numéro spécial, Quelles innovations pour quels systèmes d'élevage ? Ingrand S., Baumont R. (Eds). INRA Prod. Anim., 27 113-122.

Jago J., Eastwood C., Kerrisk K., Yule I., 2013. Precision dairy farming in Australasia: adoption, risks and opportunities. Anim. Prod. Sci., 53, 907-916.

Joly N., Gautier J.M., 2006. Pratiques de la traçabilité en élevage : des compétences à ajuster. Renc. Rech. Rum., 13, 187-190.

Jouven M., Lapeyronie P., Moulin C.H., Bocquier F., 2010. Rangeland utilization in Mediterranean farming systems. Animal 4,1746-1757.

Jouven M., Leroy H., Ickowicz A., Lapeyronie P., 2012. Can virtual fences be used to control grazing sheep? Rangeland J., 34, 111-123.

Laca E., 2009. Precision livestock production: tools and concepts. Revista Brasileira de Zootecnia., 38, 123-132.

Lai X., Liu Q., Wei X., Wang W., Zhou G., Han G., 2013. A Survey of Body Sensor Networks. Sensors, 13, 5406-5447.

Léveillé M., Jouany J.P., Brun J.B., 1979 Analyse automatique du comportement alimentaire et mérycique chez le mouton. Ann. Biol. Anim. Bioch. Biophys., 19, 889-893.

Lurette A., Trimoulard A., Bocquier B. Moulin C.H., 2012. Perceptions des éleveurs d'ovins laitiers sur les facteurs de maîtrise de la reproduction. Renc. Rech. Rum., 19, 360.

Martin G.B., Kadokawa H., 2006. «Clean, green and ethical» animal production. Case study: reproductive efficiency in small ruminants. J. Reprod. Dev., 52, 145-152.

Maton C., Bouquet P.M., Laville Y., Bocquier F., 2006. Automate de tri pour les ovins basé sur l'identification électronique. Renc. Rech. Rum., 13, 167.

Maton C., Bocquier F., Debus N., Bodin L., Teyssier J., 2010. Suivi automatisé des chaleurs et différence de saisonnalité entre brebis Texel et Mérinos dans un environnement méditerranéen. Renc. Rech. Rum., 17, 125-128.

Mercier L., Marguin L., Gauthier J.M., 2006. Atouts, contraintes et perspectives de l'identification électronique en élevage ovin. Renc. Rech. Rum., 13, 151-154.

Monod M. O., Faure P., Moiroux L., Rameau P., 2009. Stakeless fencing for mountain pastures. J. Farm Manag., 13, 697-704.

Palmer MA., Olmosb G., Boyleb L.A., Mee J.F., 2010. Estrus detection and estrus characteristics in housed and pastured Holstein-Friesian cows. Theriogenology, 74, 255-264.

Pellicer-Rubio M.T., Ferchaud S., Freret S., Tournadre H., Fatet A. Boulot S., Pavie J., Leboeuf B., Bocquier F., 2009. Les méthodes de maitrise de la reproduction disponibles chez les mammifères d'élevage et leur intérêt en agriculture biologique. INRA Prod. Anim., 22, 255-270

Peralta O.A., Pearson R.E., Nebel R.L., 2005. Comparison of three estrus detection systems during summer in a large commercial dairy herd. Anim. Reprod. Sci., 1-2, 59-72.

Perrot C., Bataille J.F., Bossis N., Caillaud D., Gallot S., Morhain B., Morin E., Roguet C., Sarzeaud P., 2013. Diversité de l'élevage français et dynamiques territoriales. Les enseignements des recensements agricoles. Renc. Rech. Rum., 20, 341-344.

Provenza F. D., Villalba J. J., 2006. Foraging in domestic herbivores: linking the internal and external milieu. In: Feeding in domestic vertebrates: from structure to behaviour. Bels V. (Ed), 210-240.

Ropert-Coudert Y., Kato A., Grémillet D., Crenner F., 2012. Bio-logging: recording the ecophysiology and behavior of animals moving freely in their environment. In: Sensors for ecology: Towards integrated knowledge of ecosystems. Le Galliard J.F., Guarini J.M., Gaill F. (Eds). Editions CNRS, Paris, ISBN : 978-29541683-0-2 Chapitre 1, 17-41.

Ruiz-Garcia L., Lunadei L., 2011. The role of RFID in agriculture: Applications, limitations and challenges. Comp. Electron. Agric., 79, 42-50.

Saumande J., 2002. Electronic detection of estrus in postpartum dairy cows: efficiency and accuracy of the $\mathrm{DEC} \mathbb{R}$ (showheat) system. Livest. Prod. Sci., 77, 265-271.

Stafford KA., Morgan ER., Coles GC., 2009. Weight-based targeted selective treatment of gastrointestinal nematodes in a commercial sheep flock. Vet. Parasitol., 164, 59-65.

Tiedemann A.R., Quigley T.M., White L.D., Lauritzen W.S., Thomas J.W., McInnis M.L. 1999. Electronic (Fenceless) Control of Livestock. Research Paper PNW-RP-510. (U.S Dept. Agricult, Forest Service, Pacific Northwest Research Station: Portland, OR.)

Umstatter C., 2011. The evolution of virtual fences: a review. Comp. Electron. Agric., 75, 10-22.

Valenza A., Giordano J.O., Lopes Jr G., Vincenti L., Amundson M.C., Fricke, P.M., 
2012. Assessment of an accelerometer system for detection of estrus and treatment with gonadotropin-releasing hormone at the time of insemination in lactating dairy cows. J. Dairy Sci., 95, 7115-7127.

Velasco-Garcia M.N., Mottram T., 2003. Biosensor Technology addressing Agricultural Problems. Biosyst. Eng., 84, 1-12.

Veysset P., Benoît M., Laignel G., Bébin D., Roulenc M., Lherm M., 2013. PAC, marchés, aléas : 23 ans d'évolution en exploitations d'élevage bovin et ovin allaitant de zones défavorisées. Et après ? Renc. Rech. Rum., 20, 345348.

Viudes G., Lurette A., Debus N., Capron J.M., Bocquier F., 2012, Détection automatisée des chaleurs chez des brebis Mérinos d'Arles pendant 4 cycles successifs. Renc. Rech. Rum., 19, 363.

Wathes C.M., Kristensen H.H., Aerts J.M. Berckmans D., 2008. Is precision livestock farming an engineer's daydream or nightmare, an animal's friend or foe, and a farmer's panacea or pitfall? Comp. Electron. Agric., 64, 2-10.

\section{Résumé}

Les fortes contraintes d'élevage dans les systèmes peu intensifiés (grands troupeaux, larges surfaces, alimentation au pâturage, voire faible productivité par animal) laissent tout de même entrevoir des formes originales d'élevage de précision. Cet élevage de précision peut notamment s'appuyer sur l'identification électronique des animaux qui permet de les reconnaitre et de les localiser. Selon que les lecteurs d'identifiants sont utilisés manuellement, à poste fixe, ou portés par un mâle, il est possible de réaliser, respectivement, des inventaires, des opérations automatisées de tri ou la détection des femelles en chaleurs. C'est au pâturage que la maîtrise du comportement des animaux est la plus délicate car un positionnement judicieux de points d'attraction des animaux (abreuvement, pierres à sel, complémentation) ne suffit pas toujours à maîtriser la répartition de la pression de pâturage pour répondre aux objectifs pastoraux et environnementaux. Dans les situations où la pose de clôtures fixes n'est pas possible ou pas souhaitable et que le gardiennage n'est pas envisageable, les clôtures virtuelles, basées sur un apprentissage comportemental spécifique des animaux, pourraient constituer une aide précieuse à l'utilisation durable des parcours. Dans ces systèmes d'élevage, la précision visée ne se situe pas à l'échelle des individus mais plutôt sur des conduites ajustées à des lots virtuels d'animaux homogènes, qui sont séparés et regroupés grâce à des dispositifs de tri efficaces. L'utilisation de systèmes automatisés de suivi du troupeau où chaque animal est identifié génère une grande quantité de données. Quels que soient les capteurs utilisés, les données recueillies doivent être stockées dans un système d'information et traitées avec des méthodes d'analyse adaptées (algorithmes, statistiques...) pour être ensuite transformées en indicateurs d'aide à la décision ou en action exécutée par des automates (porte de tri, pesées, distributions d'aliments). En alternative à des suivis individuels, une utilisation parcimonieuse des capteurs peut être envisagée pour délivrer des alertes (intrusion, attaque de prédateur, sortie d'une zone géographique définie) ; la quantité de données produites est alors minimisée. Un travail de recherche important reste à mener pour proposer des méthodes d'analyse des données et des règles de décisions pertinentes, basées sur une modélisation du comportement animal. Dans les systèmes d'élevage peu intensifiés, la place de l'éleveur est essentielle car au sein d'un grand troupeau et face à de larges surfaces de parcours les dispositifs électroniques sont à envisager comme des aides spécifiques, à intégrer dans une stratégie de conduite plus large. Notamment, il s'agit pour l'éleveur de choisir quels animaux équiper et où positionner des capteurs, relais ou clôtures virtuelles, et à choisir de le faire aux périodes critiques. Actuellement l'offre matérielle et logicielle est freinée par le manque de souplesse des solutions proposées.

\section{Abstract}

\section{Precision farming in extensive livestock systems}

Despite the strong constraints which apply to extensive livestock farming systems (large herds, large surface areas, pasture-based feeding and eventually low animal productivity), original solutions of precision farming can be developed. Precision farming can especially rely on the individual electronic identification systems (RFID) to recognize or locate the animals. Depending on the utilization of RFID-readers (either stationary or portable devices, held by an operator or carried by a male), it is possible to produce inventories, sorting operations or estrus detection. Animal behavior is most difficult to control at pasture, since the strategic placement of attraction points (water sources, salt blocks, and supplements) can prove insufficient to control the spatial distribution of the grazing pressure in a context of strong pastoral and environmental objectives. In situations where either traditional fences or shepherding cannot be implemented, virtual fences, which are based on a specific behavioral education of the animals, could contribute to a sustainable utilization of rangeland areas. In extensive livestock systems, precision farming does not apply at the individual scale, but implies adjusting management practices to virtual groups of animals sharing the same characteristics, such groups being made and unmade by efficient sorting devices. The utilization of automated herd-monitoring systems based on individual information produces a great amount of data. Whatever the type of sensor used, the data collected must be stored in an information system, then analyzed with appropriate methods (algorithms, statistics...) in order to be translated into decision-support indicators or into actions implemented by machines (sorting gate, weighing, feed distribution). As an alternative to individual monitoring, a parsimonious utilization of sensors can suffice to give alerts (intrusion, predator attack, exit from a given geographical area); the amount of data produced is thus minimized. Further research is needed to propose appropriate methods for data analysis and decision rules, based upon animal behavior modeling. In extensive farming systems, the farmer plays an essential role; when large herds and surface areas are involved, electronic devices and decision-support systems are to be considered as specific aids to be integrated in a larger management strategy. Especially, the farmer has to decide which animals to monitor, where to locate the sensors/readers or virtual fences, and when to implement precision farming, i.e. which are the critical periods. At the moment, commercial solutions for devices and software lack the versatility needed to apply to extensive systems.

BOCQUIER F., DEBUS N., LURETTE A., MATON C., VIUDES G., MOULIN C.-H., JOUVEN M., 2014. Elevage de précision en systèmes d'élevage peu intensifiés. In : Numéro spécial, Quelles innovations pour quels systèmes d'élevage ? Ingrand S., Baumont R. (Eds). INRA Prod. Anim., 27, 101-112. 